\title{
探究式教学模式在分析化学综合实验教学中的实践
}

\author{
李炜林 ${ }^{2}$, 刘红瑜 ${ }^{1, *}$, 金谷 ${ }^{2}$, 姚奇志 ${ }^{1}$, 李娇 ${ }^{1}$, 李玲玲 1 \\ 1 化学国家级实验教学示范中心(中国科学技术大学), 合肥 230026 \\ 2 中国科学技术大学化学与材料科学学院, 合肥 230026
}

\begin{abstract}
摘要: 介绍了中国科学技术大学分析化学实验教学模式的探索和实践。在掌握基本的定量分析操作的基础上, 如何 提升学生自主探究实验的能力及自主设计实验的高阶创新实验能力是分析化学实验需要达成的教学目标。探究学习 是一种以学生基于问题而展开的学习方式, 教学中我们采用这样的教学模式能充分调动学生学习的积极性, 通过实 施这样的教学模式, 学生在探究中不断提升了发现问题、分析问题、解决问题的能力, 从而达到培养学生创新能力 的目的。
\end{abstract}

关键词: 探究式教学模式; 高阶创新实验能力; 解决问题的能力

中图分类号: G64; O6

\section{Practice of Inquiry Teaching Mode in Comprehensive Experimental Teaching of Analytical Chemistry}

\author{
Weilin Li ${ }^{2}$, Hongyu Liu 1, ${ }^{1}$, Gu Jin ${ }^{2}$, Qizhi Yao ${ }^{1}$, Jiao Li ${ }^{1}$, Lingling Li ${ }^{1}$ \\ ${ }^{1}$ National Demonstration Center for Experimental Chemistry Education (University of Science and Technology of China), \\ Hefei 230026, China. \\ ${ }^{2}$ School of Chemistry and Materials Science, University of Science and Technology of China, Hefei 230026, China.
}

\begin{abstract}
This paper introduces the exploration and practice of analytical chemistry experiment teaching mode at University of Science and Technology, China. From the viewpoint of mastering the basic quantitative analysis operation, we aim to improve the students' ability to independently explore the experiment and high-level innovative experiment ability of independent design experiment in analytical chemistry experiments. Inquiry learning method is based on students' problems. In teaching, we use this teaching model to fully mobilize the students' learning enthusiasm. Through the implementation of this teaching model, students continuously improve their ability to find, analyze, and solve problems to achieve the purpose of cultivate students' innovative abilities.
\end{abstract}

Key Words: Inquiry teaching mode; High level innovation experiment ability;

Ability to find, analyze and solve problems

分析化学实验是我校化学专业本科生的必修实验课, 面对的学生是大二本科生, 学生已有的学 习基础是: 无机化学理论、无机化学实验。通过大一无机化学实验的学习, 学生了解并掌握了完成 定性化学实验的基本操作, 学生初步具有了独立开展验证性实验的能力。在此基础上, 如何进一步 提升学生定量分析的实验能力、自主探究实验的能力及自主设计实验的高阶创新实验能力是分析化

收稿: 2021-11-11; 录用: 2021-11-22; 网络发表: 2021-12-20

“通讯作者, Email: liuhyhx@ustc.edu.cn

基金资助: 中国科学技术大学教研项目(2020xjyxm038); 中国科学技术大学课程思政研究项目(2021xkcszxm02) 
学实验需要达成的教学目标。

我校分析化学实验分为I、II两种类型, 分两个学期来完成。I 型实验以定量分析为主, 让学生通 过基本的滴定分析方法掌握定量分析的基本原理、基本操作, 学会处理分析实验数据, 让学生初步 具有分析问题、解决问题的能力。II型实验以研究型探究实验和创新设计实验为主, 研究型探究实验 侧重培养学生的科研思维和小组协作能力, 创新设计实验则更加侧重学生自主设计、开展实验课题 的能力, 让学生在模拟科研的情境下完成一次科研体验 ${ }^{[1-4]}$ 。

\section{1 做好、II型实验的衔接}

如何做好I、II型实验的衔接, 关键是如何完成从基础实验到综合实验乃至自主设计实验的教学 模式转变 (表1 为 $I 、 I I$ 型实验内容)。I型实验前七个实验都是验证型实验, 第八个实验是综合探究实验、 第九个是自主设计实验, 这两个实验都是为II型实验的开展做铺垫, 如何做好两个不同层次的实验 的衔接是需要在教学中深入探索的问题。

表1 I、II型实验内容

\begin{tabular}{|c|c|c|c|}
\hline \multicolumn{2}{|r|}{ I型 } & \multicolumn{2}{|r|}{ II型 } \\
\hline \multicolumn{2}{|c|}{ 基本操作、数据处理、实验习惯 } & \multicolumn{2}{|r|}{ 科研创新能力 } \\
\hline 实验一 & 基本操作和安全教育 & 实验一 & 十二烷基硫酸钠(SDS)在 $\mathrm{Al}_{2} \mathrm{O}_{3}$ 表面的自组装、表征及用于铜离子的 \\
\hline 实验二 & 未知碱的测定 & & 分离、富集和测定 \\
\hline 实验三 & 有机酸摩尔质量测定 & 实验二 & 海藻酸盐微胶囊的制备及应用探究 \\
\hline 实验四 & 返滴定法测定明矾中的铝 & 实验三 & 碘与水溶性聚合物显色反应研究和分析方法的建立 \\
\hline 实验五 & 碘量法测定铜合金中的铜 & 实验四 & 绿色液-固萃取体系分离钴和镍的机理研究 \\
\hline 实验六 & 无录盐法测定铁矿石中的铁 & 实验五 & 含铬废水的绿色处理方法研究 \\
\hline 实验七 & 高锰酸钾法测定白云石中的钙 & & \\
\hline 实验八 & 硫酸钡均相沉淀历程探究 & & \\
\hline 实验九/十 & 自主设计实验 & & \\
\hline
\end{tabular}

验证型实验都是基于已知实验条件, 学生按部就班地开展实验就可以, 因此课堂教学还是以传 统的启发式讲授为主、基于问题的研讨式为辅的教学模式(表2)。这样的教学模式并不适合综合探究 式实验教学, 因为探究式实验是在基于已知的基本原理和实验目标的基础上, 学生需要自主设计实 验条件, 基于实验数据的分析, 不断优化实验条件的设计和选取。因此需要侧重课前的文献调研, 课中更应该以翻转课堂的探究式教学模式来让学生成为课堂的主角(表3)。

\section{表2 I型基础实验教学模式}

\begin{tabular}{clllll}
\hline 实验编号 & \multicolumn{1}{c}{ 实验项目 } & \multicolumn{1}{c}{ 重点与难点 } & 实验类型 & 学时 & 课堂教学模式 \\
\hline 1 & 基本操作和安全教育(验证型实验) & 移液管、滴定管的规范操作 & 验证型 & 8 学时 & 讲授、演示、实操 \\
2 & 未知碱的测定(验证型实验) & 分析天平称量操作、容量瓶定容 & 验证型 & 8 学时 & 讲授、演示、实操 \\
3 & 有机酸摩尔质量测定(验证型实验) & 滴定终点判断 & 验证型 & 8 学时 & 讲授、演示、实操 \\
4 & 返滴定法测定明矾中的铝(验证型 & 样品处理、 $\mathrm{pH}$ 控制 & 验证型 & 8 学时 & 讲授、演示、实操 \\
& 实验) & & & & \\
5 & 碘量法测定铜合金中的铜(验证型 & 碘的氧化和挥发的控制 & 验证型 & 8 学时 & 讲授、演示、实操 \\
& 实验) & & & & \\
\hline
\end{tabular}


大学化 学 Univ. Chem. 2022, 37 (4), 2111038 (3 of 6)

(续表2)

\begin{tabular}{|c|c|c|c|c|c|}
\hline 实验编号 & 实验项目 & 重点与难点 & 实验类型 & 学时 & 课堂教学模式 \\
\hline \multirow[t]{2}{*}{6} & 无永盐法测定铁矿石中的铁(验证 & 亚锡离子量的控制、防止亚铁离 & 验证型 & 8 学时 & 讲授、演示、实操 \\
\hline & 型实验) & 子被氧气氧化 & & & \\
\hline \multirow[t]{2}{*}{7} & 高锰酸钾法测定白云石中的钙(综 & 晶形沉淀的制备、分离 & 综合型 & 8 学时 & 讲授、演示、实操 \\
\hline & 合实验) & & & & \\
\hline \multirow[t]{2}{*}{8} & 硫酸钡均相沉淀历程探究(综合探 & 通过正交实验条件的设计探究 & 综合探究型 & 8 学时 & 讨论、演示、实操探究 \\
\hline & 究实验) & 反应机理 & & & \\
\hline \multirow[t]{2}{*}{$9 / 10$} & 自主设计实验(设计实验) & 设计方案的可行性 & 设计型 & 16 学时 & 学生汇报设计报告、教师 \\
\hline & & & & & 同行点评、实操、结果汇报 \\
\hline
\end{tabular}

表3 II型综合实验教学模式

\begin{tabular}{|c|c|c|c|c|c|}
\hline 编号 & 实验项目 & 重点与难点 & 实验类型 & 学时 & 课堂教学模式 \\
\hline \multirow[t]{2}{*}{1} & 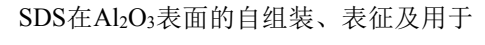 & 自组装原理 & 综合探究型 & 16 学时 & 讨论、演示、实操、总结 \\
\hline & 铜离子的分离、富集和测定 & & & & 分析、进一步探究 \\
\hline \multirow[t]{2}{*}{2} & 海藻酸盐微胶囊的制备及应用探究 & 微胶囊的可控制备 & 综合探究型 & 16 学时 & 讨论、演示、实操、总结 \\
\hline & & & & & 分析、进一步探究 \\
\hline \multirow[t]{2}{*}{3} & 碘与水溶性聚合物显色反应研究和分析 & 定量分析方法的建立 & 综合探究型 & 16 学时 & 讨论、演示、实操、总结 \\
\hline & 方法的建立 & & & & 分析、进一步探究 \\
\hline \multirow[t]{2}{*}{4} & 绿色液-固萃取体系分离钴和镍的机理 & 液固萃取机理 & 综合探究型 & 16 学时 & 讨论、演示、实操、总结 \\
\hline & 研究 & & & & 分析、进一步探究 \\
\hline \multirow[t]{2}{*}{5} & 含铬废水的绿色处理方法研究 & 设计方案的可行性、 & 设计型 & 16 学时 & 汇报设计报告、教师同行 \\
\hline & & 绿色化 & & & 点评、实操、结果汇报 \\
\hline
\end{tabular}

\section{2 以“SDS在 $\mathrm{Al}_{2} \mathrm{O}_{3}$ 表面的自组装、表征及用于铜离子的分离、富集和测定” 实验为例}

表面活性剂自组装形成的分子有序组合体，属于超分子领域，是众多研究领域的热点，该实验 项目被选为国家级实验教学示范中心示范实验项目。未改性的氧化铝虽可吸附金属离子, 但吸附效 率很差, 难以应用, 通过对其表面改性, 可以提高其吸附效率。通过调节溶液的 $\mathrm{pH}$ 使氧化铝表面带 正电, 这样就可以和阴离子表面活性剂十二烷基硫酸钠(SDS)通过静电作用实现对氧化铝的表面改 性。改性后的氧化铝表面变为疏水性, 所以难以直接吸附铜离子。因此需要对铜离子也进行处理, 通过二乙基氨基二硫代甲酸钠(铜试剂)和铜离子作用形成弱极性的络合物, 就非常容易吸附在氧化 铝表面的SDS栅栏层中。如果有干扰离子存在, 则通过调节溶液的pH来调控金属离子和铜试剂的结 合能力, 从而达到分离的目的。该体系不仅可以分离、富集铜离子, 也可用于处理废水中的其他重 金属离子, 分离效果通过分光光度法就可以进行表征。该实验是非常典型的前沿综合探究实验项目, 涉及到了很多学生在理论课上并没有学到的知识, 实验原理及流程如图1所示。

本实验分为两次, 第一次是在给定实验条件下开展实验(改性时 $\mathrm{pH}$ 为 2 , 测定铜离子标准曲线时 $\mathrm{pH}$ 为4), 如图2所示。通过第一次实验探究, 学生发现实验过程中有很多异常现象, 基于实验原理并结 合这些异常实验现象, 我们鼓励并引导学生在第一次实验的基础上进行进一步探究。比如针对以下 实验条件及实验中的异常实验现象进行了探究。

(1) 在进行最后一步铜离子的吸附和分析实验时, 出现样品的吸光度为负的情况, 我们并没有 直接告诉学生是什么原因, 而是引导学生结合分光光度法的原理进行思考, 学生通过查阅资料分析, 


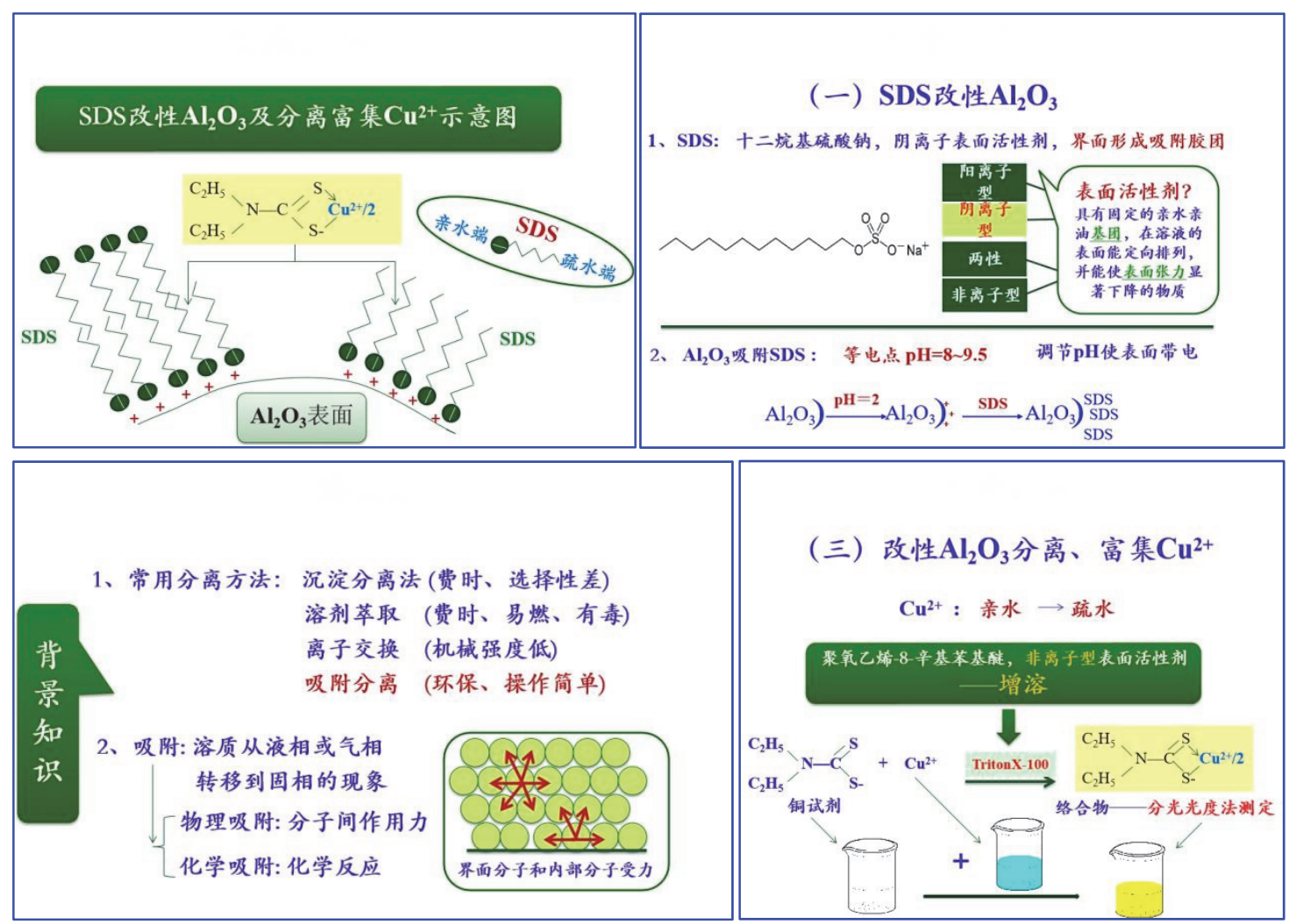

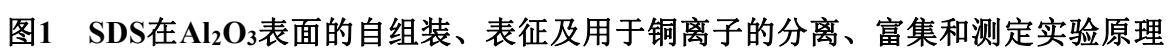

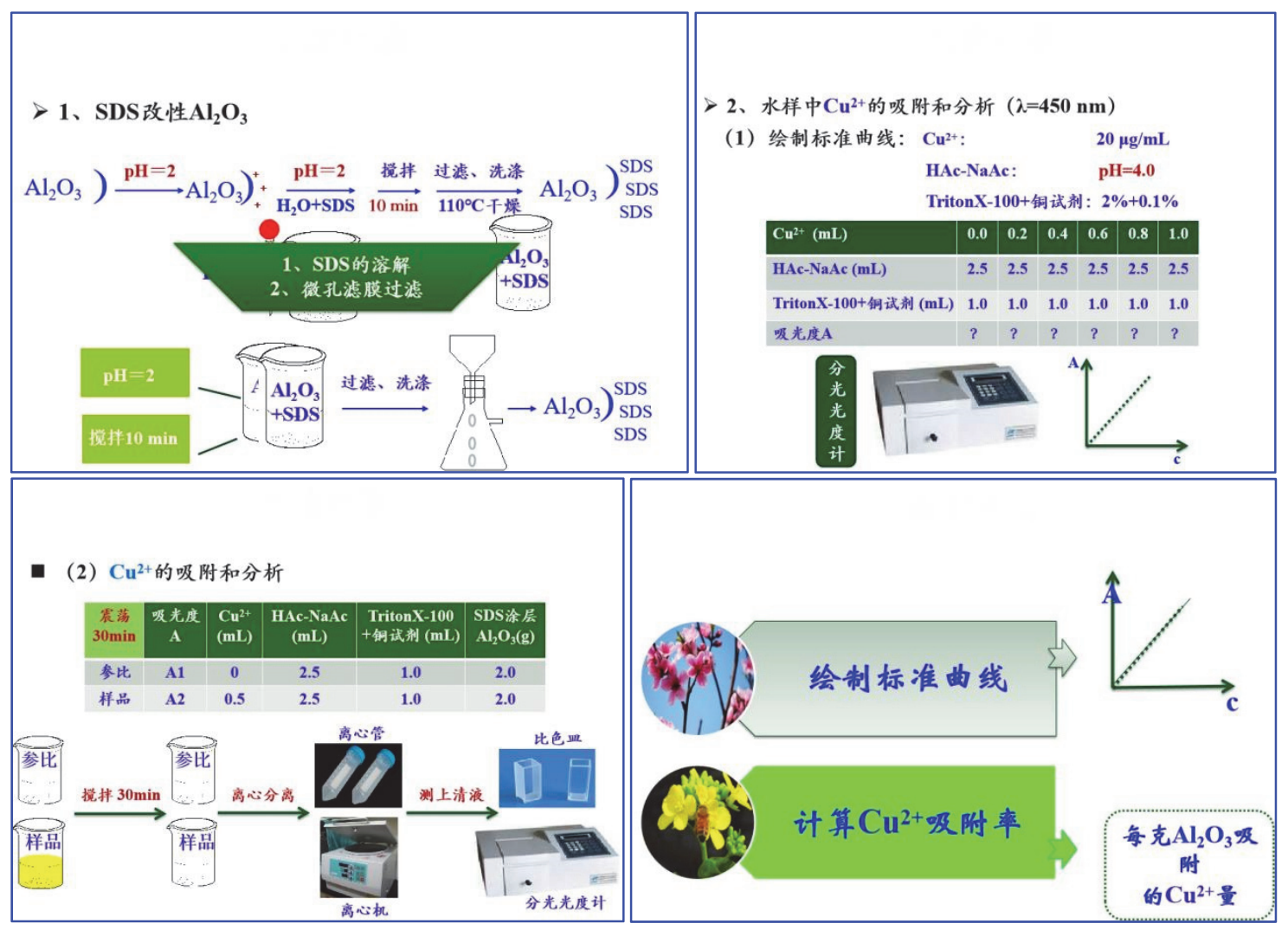

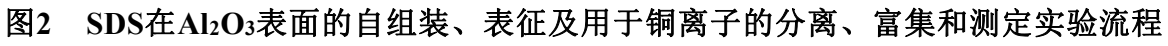


造成这种情况的主要原因为: 在吸光度测量过程中, 空白组存在气泡而导致吸光度增大, 造成空白 组的吸光度比样品组的大; 离心分离时有部分氧化铝没有离心下来, 造成空白组的吸光度比样品组 的大; 样品中, 铜离子和铜试剂络合消耗了一定量的铜试剂, 而参比中并没有铜离子, 因此参比的 吸光度可能大于样品的。就第三种原因, 学生将参比换为绘制标准曲线中的第二份溶液(加 $0.2 \mathrm{~mL}$ 铜 离子标准溶液), 再次进行测定, 吸光度值变为正值 0.006 了。

(2) 在绘制铜离子吸光度标准曲线过程中出现问题(比如出现负值、浓度较大的溶液吸光度反而 较小等问题), 虽然重配后绘制出了曲线, 但也可能还存在较大误差。考虑到各组实验使用的缓冲溶 液都是各自配制, pH也会存在一定差异, 而在配制标准溶液时就会使用到缓冲溶液, 那么溶液的 $\mathrm{pH}$ 是否会影响溶液的吸光度呢? 为此, 学生设计了一系列不同 $\mathrm{pH}$ 条件下的铜离子标准曲线测定实验, 以此来考查 $\mathrm{pH}$ 对标准曲线的影响。该实验第一次实验时绘制出的标准曲线可以作为一组数据充当对 照, 分别在配制标准溶液时加入酸、碱、蒸馏水测定吸光度, 得到新的标准曲线与之比较。最后通 过探究实验发现, 随着加入的缓冲溶液的 $\mathrm{pH}$ 逐渐升高, 溶液的吸光度逐渐增大, 线性关系都挺好(如 表4所示), 铜试剂为二乙基二硫代氨基甲酸钠, 化学式为 $\left(\mathrm{C}_{2} \mathrm{H}_{5}\right)_{2} \mathrm{NCSSNa}$, 其水溶液呈碱性, 遇酸会 分解, 因此当溶液的 $\mathrm{pH}$ 较低时不利于铜试剂的稳定性。

表4 $\mathrm{pH}$ 对 $\mathrm{Cu}^{2+}$ 标准曲线的影响

\begin{tabular}{cccc}
\hline \multirow{2}{*}{$V\left(\mathrm{Cu}^{2+}\right.$ 标液 $) / \mathrm{mL}$} & \multicolumn{3}{c}{ 吸光度 $A$} \\
\cline { 2 - 4 } & $\mathrm{pH}=2.65$ & $\mathrm{pH}=3.34$ & $\mathrm{pH}=4.44$ \\
\hline 0 & 0 & 0 & 0 \\
0.2 & 0.013 & 0.057 & 0.059 \\
0.4 & 0.022 & 0.103 & 0.112 \\
0.6 & 0.03 & 0.156 & 0.16 \\
0.8 & 0.045 & 0.193 & 0.212 \\
\hline
\end{tabular}

(3) 改性 $\mathrm{pH}$ 对吸附铜离子效率的影响: 该实验的原理是利用改性后的 $\mathrm{Al}_{2} \mathrm{O}_{3}$ 表面的栅栏层吸附铜 试剂的配合物, 从而达到集聚、分离、富集铜离子的目的。对 $\mathrm{Al}_{2} \mathrm{O}_{3}$ 改性效果直接影响到铜离子最终 吸附及分离的效率。又因在实验过程中改性溶液 $\mathrm{pH}$ 均在 1 左右, 而已知 $\mathrm{Al}_{2} \mathrm{O}_{3}$ 在 $\mathrm{pH}=2$ 的溶液环境下 改性效果最好, 故通过设计一系列 $\mathrm{pH}$ 梯度来探究 $\mathrm{Al}_{2} \mathrm{O}_{3}$ 改性效果及最后的吸附结果。探究结果如表 5 所示, 可见 $\mathrm{pH} 2.22$ 左右吸附效率最高, 这也可以从实验原理来进行解释, SDS 是一种阴离子型表面 活性剂, 如果pH过低, SDS会结合氢离子形成对应的酸, 导致其无法和 $\mathrm{Al}_{2} \mathrm{O}_{3}$ 作用, 使得改性效果变 差, 从而降低了对铜离子的吸附效率; 当 $\mathrm{pH}$ 过高时, $\mathrm{Al}_{2} \mathrm{O}_{3}$ 表面带的正电荷过少, 从而减弱了其和 SDS 的作用, 进而降低了对铜离子的吸附效率。

表5 改性 $\mathrm{pH}$ 对 $\mathrm{Cu}^{2+}$ 吸附效率的影响

\begin{tabular}{cc}
\hline $\mathrm{pH}$ & 吸附后溶液的吸光度 $A$ \\
\hline 1 & 0.002 \\
1.45 & 0.005 \\
2.22 & -0.016 \\
3 & 0.01 \\
\hline
\end{tabular}

\section{3 结语}

探究学习是一种通过学生在实验过程中发现问题后对实验进行拓展探究的学习方式, 教学中我 们采用这样的教学模式能充分调动学生学习的积极性, 通过实施这样的教学模式, 学生在探究中不 
断提升了发现问题、分析问题、解决问题的能力, 而创新能力的培养也是遵循这样的规律。这也充 分体现了教师的 “导” 是充分为学生的 “学” 服务的教学理念。因此为了达到培养学生创新能力的 目的, 在化学实验教学中开展探究式教学模式非常有必要也非常有效 $[5,6]$ 。

\section{参 考 文 献}

[1] 刘红瑜, 张晨龙, 江伟韬, 刘立岩, 邢伟龙, 金谷, 姚奇志, 李娇, 王晓葵, 李玲玲. 大学化学, 2020, 35 (2), 33.

[2] 刘红瑜, 原思浩, 金谷. 大学化学, 2021, 36 (2), 1912074.

[3] 刘红瑜, 郭昕辉, 陈俊丞, 宋奥博, 金谷, 姚奇志, 李娇, 李玲玲. 大学化学, 2020, 35 (12), 221.

[4] 李娇, 金谷, 姚奇志, 李玲玲, 刘红瑜. 大学化学, 2021, 36 (6), 2008019.

[5] 董立军, 王薇, 吕东显, 兰景风. 大学化学, 2021, 36 (9), 2105056.

[6] 王静, 李会平, 王小燕, 罗世忠, 王书文. 大学化学, 2021, 36 (4), 2005005. 